\title{
A Fast Algorithm for 2-D Direction-of-Arrival Estimation
}

\author{
Yuntao $\mathrm{Wu}^{1}$, Guisheng Liao ${ }^{1}$ and H. C. So ${ }^{2}$ \\ ${ }^{1}$ Laboratory for Radar Signal Processing, Xidian University, Xian, China \\ ${ }^{2}$ Department of Computer Engineering \& Information Technology, City University of Hong Kong, \\ Tat Chee Avenue, Kowloon, Hong Kong \\ Tel : (852) $27887780 \quad$ Fax : (852) $27888292 \quad$ email: ithcso@cityu.edu.hk
}

April 23, 2003

Keywords : 2-D DOA estimation, propagator method, fast algorithm

Abstract : A computationally efficient method for two-dimensional direction-of-arrival estimation of multiple narrowband sources impinging on the far field of a planar array is presented. The key idea is to apply the propagator method which only requires linear operations but does not involve any eigendecomposition or singular value decomposition as in common subspace techniques such as MUSIC and ESPRIT. Comparing with a fast ESPRIT-based algorithm, it has a lower computational complexity particularly when the ratio of array size to the number of sources is large, at the expense of negligible performance loss. Simulation results are included to demonstrate the performance of the proposed technique.

\section{Introduction}

The problem of estimating the two-dimensional (2-D) directions-of-arrival (DOAs), namely, the azimuth and elevation angles, of multiple sources has received considerable attention in the field of array processing [1]-[7]. Although the maximum likelihood estimator [1] provides optimum parameter estimation, its computational complexity is extremely demanding. Simpler but suboptimal solutions can be achieved by the subspace based approach, which relies on the decomposition of the observation space into signal subspace and noise subspace. However, conventional subspace techniques for 2-D DOA estimation such as MUSIC [2] and ESPRIT [3]-[7] necessitate eigendecomposition of the sample covariance matrix (SCM) or the singular value decomposition (SVD) of the data matrix (DM) to estimate the signal and noise subspaces, and huge computation will be involved particularly when the dimensions of the underlying matrices are large, for example, in the case of large towed arrays in sonar. Furthermore, many of the above methods [1]-[6] require reasonably accurate initial DOA estimates, 2-D search and/or complex pair matching of the azimuth and elevation angles, although the ESPRIT-based technique proposed in [7] is direct, that is, free of these operations. 
The aim of this paper is to develop a computationally simple 2-D DOA estimation algorithm by utilizing the propagator method (PM) [8], which is also a subspace scheme but does not involve eigendecomposition or SVD. In fact, [9] has proposed a PM-based DOA estimation method but unfortunately, a 2-D peak search is needed. Our derived algorithm is direct and has a lower computational complexity comparing with [7], particularly when the ratio of the number of sensors to the number of sources is large, at the expense of negligible performance loss.

The rest of the paper is organized as follows. The data model is presented in Section 2. Our 2-D DOA estimation algorithm is developed in Section 3. In Section 4, simulation results are included to demonstrate the effectiveness of the proposed method, while in Section 5, conclusions are drawn.

\section{Data Model}

Consider two parallel uniform linear arrays (ULAs) with interelement spacing equals $d$, such that one ULA consists of $(N+1)$ sensors while another has $N$ sensors, which is depicted in Figure 1. From this configuration, we form three sub-ULAs of $N$ sensors, namely, $X$, $Y$ and $Z$, which have coordinates $(d \cdot i, 0),(d \cdot i+d, 0)$ and $(d \cdot i, d)$, for $0 \leq i \leq N-1$, respectively. As in common ESPRIT-based approach [6], it is necessary to have 3 subarrays for 2-D DOA estimation, while one-dimensional DOA estimation problem only requires two subarrays. It is noteworthy that although a configuration of two ULAs is considered, our proposed method can be extended to other planar array geometries [6]-[7]. Suppose that there are $p$ narrow-band sources, $\mathbf{s}(t)$, with same wavelength $\lambda$ impinging on the arrays, such that the $k$ th source has an elevation angle $\theta_{k}$ and azimuth angle $\phi_{k}$. The observed signals at the $X, Y$ and $Z$ subarrays are given by

$$
\begin{gathered}
\mathbf{x}(t)=\mathbf{A} \mathbf{s}(t)+\mathbf{n}_{x}(t) \\
\mathbf{y}(t)=\mathbf{A} \boldsymbol{\Phi}_{y} \mathbf{s}(t)+\mathbf{n}_{y}(t) \\
\mathbf{z}(t)=\mathbf{A} \boldsymbol{\Phi}_{z} \mathbf{s}(t)+\mathbf{n}_{z}(t), \quad t=1,2, \cdots, M
\end{gathered}
$$

respectively, where each sensor receives $M$ snapshots. The matrices and vectors in (1) have the following forms:

$$
\begin{aligned}
& \mathbf{x}(t)=\left[\begin{array}{lll}
x_{1}(t) & x_{2}(t) \cdots x_{N}(t)
\end{array}\right]^{T} \\
& \mathbf{y}(t)=\left[\begin{array}{lll}
y_{1}(t) & y_{2}(t) \cdots y_{N}(t)
\end{array}\right]^{T} \\
& \mathbf{z}(t)=\left[\begin{array}{lll}
z_{1}(t) & z_{2}(t) \cdots z_{N}(t)
\end{array}\right]^{T} \\
& \mathbf{s}(t)=\left[\begin{array}{ll}
s_{1}(t) & s_{2}(t) \cdots s_{p}(t)
\end{array}\right]^{T} \\
& \mathbf{n}_{k}=\left[\begin{array}{ll}
n_{k, 1}(t) & n_{k, 2}(t) \cdots n_{k, N}(t)
\end{array}\right]^{T}, \quad k=x, y, z \\
& \mathbf{A}=\left[\mathbf{a}_{1}\left(\theta_{1}, \phi_{1}\right) \mathbf{a}_{2}\left(\theta_{2}, \phi_{2}\right) \cdots \mathbf{a}_{p}\left(\theta_{p}, \phi_{p}\right)\right] \\
& \mathbf{a}_{i}\left(\theta_{i}, \phi_{i}\right)=\left[\begin{array}{ll}
1 & e^{j 2 \pi \frac{d}{\lambda} \cos \left(\theta_{i}\right) \sin \left(\phi_{i}\right)} \cdots e^{j 2 \pi(N-1) \frac{d}{\lambda} \cos \left(\theta_{i}\right) \sin \left(\phi_{i}\right)}
\end{array}\right]^{T}, \quad i=1,2, \cdots, p \\
& \boldsymbol{\Phi}_{y}=\operatorname{diag}\left(e^{j 2 \pi \frac{d}{\lambda} \cos \left(\theta_{1}\right) \sin \left(\phi_{1}\right)}, e^{j 2 \pi \frac{d}{\lambda} \cos \left(\theta_{2}\right) \sin \left(\phi_{2}\right)}, \cdots, e^{j 2 \pi \frac{d}{\lambda} \cos \left(\theta_{p}\right) \sin \left(\phi_{p}\right)}\right) \\
& \boldsymbol{\Phi}_{z}=\operatorname{diag}\left(e^{j 2 \pi \frac{d}{\lambda} \sin \left(\theta_{1}\right) \sin \left(\phi_{1}\right)}, e^{j 2 \pi \frac{d}{\lambda} \sin \left(\theta_{2}\right) \sin \left(\phi_{2}\right)}, \cdots, e^{j 2 \pi \frac{d}{\lambda} \sin \left(\theta_{p}\right) \sin \left(\phi_{p}\right)}\right)
\end{aligned}
$$

where $T$ denotes the transpose operation. It is assumed that the source number $p$, with $p<N$, is known a priori and all sources are not fully correlated while the additive noises $\mathbf{n}_{x}(t), \mathbf{n}_{y}(t)$ and $\mathbf{n}_{z}(t)$ are stationary zero-mean random processes which are uncorrelated with $\mathbf{s}(t)$. We further assume that the columns of $\mathbf{A}$ are linearly independent and the 2-D angle pairs $\left(\theta_{i}, \phi_{i}\right)$ are distinct points in the $[0, \pi / 2] \times[0,2 \pi]$ plane. Given $\mathbf{x}(t), \mathbf{y}(t)$ and $\mathbf{z}(t)$, the task is to estimate $\left(\theta_{i}, \phi_{i}\right), i=1,2, \cdots, p$. In our proposed method, we first find the matrices $\boldsymbol{\Phi}_{y}$ and $\boldsymbol{\Phi}_{z}$, and the 2-D angles can then be obtained easily from their diagonal elements. 


\section{Proposed Method}

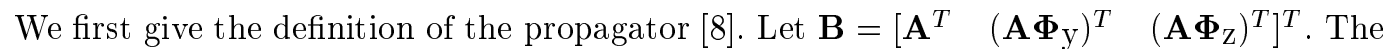
matrix $\mathbf{A}$ is partitioned into $\mathbf{A}=\left[\begin{array}{ll}\mathbf{A}_{1}^{T} & \mathbf{A}_{2}^{T}\end{array}\right]^{T}$, where $\mathbf{A}_{1}$ and $\mathbf{A}_{2}$ contain the 1 th to $p$ th rows and the $(p+1)$ th to $N$ th rows of the matrix $\mathbf{A}$, respectively. Similarly, we partition $\mathbf{B}$ as $\mathbf{B}=\left[\begin{array}{ll}\mathbf{A}_{1}^{T} & \mathbf{B}_{2}^{T}\end{array}\right]^{T}$ where $\mathbf{B}_{2}$ comprises the last $(p+1)$ th to $3 N$ th rows of $\mathbf{B}$. Under the hypothesis that $\mathbf{A}_{1}$ is nonsingular, the propagator $\mathbf{P}$ is defined as a unique linear operator of the form

$$
\mathbf{P}^{H} \mathbf{A}_{1}=\mathbf{B}_{2}
$$

where $H$ denotes the Hermitian transpose.

Grouping the sensor output as $\mathbf{q}(t)=\left[\begin{array}{llll}\mathbf{x}^{T}(t) & \mathbf{y}^{T}(t) & \mathbf{z}^{T}(t)\end{array}\right]^{T}$, the propagator can be estimated from the DM, namely $\mathbf{Q}=[\mathbf{q}(1), \mathbf{q}(2), \cdots, \mathbf{q}(M)]$ or the SCM, viz $\mathbf{R}=$ $\frac{1}{M} \sum_{t=1}^{M} \mathbf{q}(t) \mathbf{q}^{H}(t)$, as follows. Partitioning $\mathbf{Q}$ and $\mathbf{R}$ into

$$
\mathbf{Q}=\left[\begin{array}{l}
\mathbf{Q}_{1} \\
\mathbf{Q}_{2}
\end{array}\right] \quad \text { and } \quad \mathbf{R}=\left[\begin{array}{ll}
\mathbf{R}_{1} & \mathbf{R}_{2}
\end{array}\right]
$$

where $\mathbf{Q}_{1}$ and $\mathbf{Q}_{2}$ contain the first $p$ and last $(3 N-p)$ rows of $\mathbf{Q}$ while $\mathbf{R}_{1}$ and $\mathbf{R}_{2}$ contain the first $p$ and last $(3 N-p)$ columns of $\mathbf{R}$. The least squares solutions for the propagator based on DM and SCM are given by [8]

$$
\hat{\mathbf{P}}_{\mathrm{DM}}=\left(\mathbf{Q}_{1} \mathbf{Q}_{1}^{H}\right)^{-1} \mathbf{Q}_{1} \mathbf{Q}_{2}^{H} \quad \text { and } \quad \hat{\mathbf{P}}_{\mathrm{SCM}}=\left(\mathbf{R}_{1}^{H} \mathbf{R}_{1}\right)^{-1} \mathbf{R}_{1}^{H} \mathbf{R}_{2}
$$

From the partitioning of $\mathbf{A}$ and $\mathbf{B}$, it is easily seen that

$$
\mathbf{B}_{2}^{T}=\left[\mathbf{A}_{2}^{T}\left(\mathbf{A}_{1} \boldsymbol{\Phi}_{y}\right)^{T}\left(\mathbf{A}_{2} \boldsymbol{\Phi}_{y}\right)^{T}\left(\mathbf{A}_{1} \boldsymbol{\Phi}_{z}\right)^{T}\left(\mathbf{A}_{2} \boldsymbol{\Phi}_{z}\right)^{T}\right]^{T}
$$

On the other hand, we can partition $\hat{\mathbf{P}}^{H}$, which denotes the Hermitian transpose of either $\hat{\mathbf{P}}_{\mathrm{DM}}$ or $\hat{\mathbf{P}}_{\mathrm{SCM}}$, as

$$
\hat{\mathbf{P}}^{H}=\left[\begin{array}{lllll}
\hat{\mathbf{P}}_{1}^{T} & \hat{\mathbf{P}}_{2}^{T} & \hat{\mathbf{P}}_{3}^{T} & \hat{\mathbf{P}}_{4}^{T} & \hat{\mathbf{P}}_{5}^{T}
\end{array}\right]^{T}
$$

where the dimensions of $\mathbf{A}_{2}, \mathbf{A}_{1} \boldsymbol{\Phi}_{y}, \mathbf{A}_{2} \boldsymbol{\Phi}_{y}, \mathbf{A}_{1} \boldsymbol{\Phi}_{z}$ and $\mathbf{A}_{2} \boldsymbol{\Phi}_{z}$ are identical to those of $\hat{\mathbf{P}}_{1}$, $\hat{\mathbf{P}}_{2}, \hat{\mathbf{P}}_{3}, \hat{\mathbf{P}}_{4}$ and $\hat{\mathbf{P}}_{5}$, respectively.

According to (3) and comparing (6) and (7), we develop the following equations:

$$
\begin{aligned}
\hat{\mathbf{P}}_{1} \mathbf{A}_{1} & =\mathbf{A}_{2} \\
\hat{\mathbf{P}}_{3} \mathbf{A}_{1} & =\mathbf{A}_{2} \boldsymbol{\Phi}_{y} \\
\hat{\mathbf{P}}_{5} \mathbf{A}_{1} & =\mathbf{A}_{2} \boldsymbol{\Phi}_{z}
\end{aligned}
$$

From (8) and assuming that $N>2 p$, we get

$$
\mathbf{A}_{1}=\hat{\mathbf{P}}_{1}^{\#} \mathbf{A}_{2}
$$

where \# denotes the pseudoinverse. Substituting (11) into (9) and (10) yields

$$
\begin{aligned}
& \hat{\mathbf{P}}_{3} \hat{\mathbf{P}}_{1}^{\#} \mathbf{A}_{2}=\mathbf{A}_{2} \boldsymbol{\Phi}_{y} \\
& \hat{\mathbf{P}}_{5} \hat{\mathbf{P}}_{1}^{\#} \mathbf{A}_{2}=\mathbf{A}_{2} \boldsymbol{\Phi}_{z}
\end{aligned}
$$


This implies that the estimates of the diagonal elements of matrices $\boldsymbol{\Phi}_{y}$ and $\boldsymbol{\Phi}_{z}$ can be obtained by the $p$ eigenvalues of $\hat{\mathbf{P}}_{3} \hat{\mathbf{P}}_{1}^{\#}$ and $\hat{\mathbf{P}}_{5} \hat{\mathbf{P}}_{1}^{\#}$, respectively, using eigenvalue factorization:

$$
\begin{aligned}
\hat{\mathbf{P}}_{3} \hat{\mathbf{P}}_{1}^{\#} & =\mathbf{U}_{1} \hat{\boldsymbol{\Phi}}_{y} \mathbf{U}_{1}^{-1} \\
\hat{\mathbf{P}}_{5} & \hat{\mathbf{P}}_{1}^{\#}=\mathbf{U}_{2} \hat{\boldsymbol{\Phi}}_{z} \mathbf{U}_{2}^{-1}
\end{aligned}
$$

where $\mathbf{U}_{1}$ and $\mathbf{U}_{2}$ are the corresponding eigenvector matrices while $\hat{\boldsymbol{\Phi}}_{y}=$ $\operatorname{diag}\left(\hat{\phi}_{y, 1}, \hat{\phi}_{y, 2}, \cdots, \hat{\phi}_{y, p}\right)$ and $\hat{\boldsymbol{\Phi}}_{z}=\operatorname{diag}\left(\hat{\phi}_{z, 1}, \hat{\phi}_{z, 2}, \cdots, \hat{\phi}_{z, p}\right)$ are the estimates of $\boldsymbol{\Phi}_{y}$ and $\boldsymbol{\Phi}_{z}$. As a result, the estimated elevation and azimuth angles are computed from

$$
\begin{aligned}
& \hat{\theta}_{i}=\tan ^{-1}\left(\angle\left(\frac{\hat{\phi}_{z, i}}{\hat{\phi}_{y, i}}\right)\right) \\
& \hat{\phi}_{i}=\sin ^{-1}\left(\frac{1}{2 \pi} \frac{\lambda}{d} \sqrt{\left(\angle\left(\hat{\phi}_{y, i}\right)\right)^{2}+\left(\angle\left(\hat{\phi}_{z, i}\right)\right)^{2}}\right), \quad i=1,2, \cdots, p
\end{aligned}
$$

We also notice that the eigenvectors in $\mathbf{U}_{1}$ and $\mathbf{U}_{2}$ can be considered as the estimates of the $p$ column components of the matrix $\mathbf{A}_{2}$. By contrasting these two sets of eigenvectors, it is easy [6] to determine the correct pairs of $\left(\hat{\theta}_{i}, \hat{\phi}_{i}\right)$. It is noteworthy that for $2 p \geq N>p$, we can use the equations $\hat{\mathbf{P}}_{2} \mathbf{A}_{1}=\mathbf{A}_{1} \boldsymbol{\Phi}_{y}$ and $\hat{\mathbf{P}}_{4} \mathbf{A}_{1}=\mathbf{A}_{1} \boldsymbol{\Phi}_{z}$, instead of (8)-(10).

Regarding major computational complexity, the number of multiplications involved in calculating $\mathbf{P}$ is in the order of $3 N M p$, that is, $O(3 N M p)$. Comparing with the direct ESPRITbased technique [7] which requires $O\left(N^{3}+3 N^{2} M\right)$ in the eigendecomposition of the cross covariance matrices, we see under typical circumstances that $M>>N$, the ratio of the computational requirement of the proposed technique to that of [7] is $O(p / N)$. This implies when the array size is large and/or the number of sources is small, it is advantageous to use the former method for saving computations.

\section{Simulation Results}

Computer simulations have been conducted to evaluate the 2-D DOA estimation performance of the proposed method. Comparison with the direct 2-D ESPRIT algorithm [7] is also made. The sensor displacement $d$ is taken to be half the wavelength of the signal waves. We consider two uncorrelated signal sources with identical powers while the additive noises are white Gaussian processes. The number of snapshots at each sensor is $M=200$. We use root mean square error (RMSE), which is defined as $\sqrt{E\left\{\left(\hat{\theta}_{i}-\theta_{i}\right)^{2}+\left(\hat{\phi}_{i}-\phi_{i}\right)^{2}\right\}}, i=1,2$, as the performance measure. All results provided are based on 500 independent runs.

In the first test, each subarray consists of $N=16$ sensors which correspond to a total number of 33 sensors. Figure 2 plots the RMSEs for the first signal source of the proposed and direct 2-D ESPRIT methods as a function of signal-to-noise ratio (SNR). The actual 2 -D DOAs of the first and second sources are given by $\left(30^{\circ}, 60^{\circ}\right)$ and $\left(60^{\circ}, 30^{\circ}\right)$, respectively. We see that the proposed method is comparable to [7] and the performance degradation of the former is only around $0.5 \mathrm{~dB}$, because the effective aperture size in the latter is larger. Although not shown, the RMSEs for the second source are very similar to those in Figure 2. The above test is repeated for different $N$ at SNR $=20 \mathrm{~dB}$ and the corresponding RMSEs are plotted in Figure 3. Again, we have not included the results for the second source because of similarity to Figure 3 . It can be observed that the estimation performance of the proposed algorithm is close to that of the 2-D ESPRIT method when $N$ is sufficiently large. 


\section{Conclusions}

A fast algorithm for 2-D DOA estimation is proposed based on the propagator method. The main motivation of using the propagator is that it only requires linear operations but does not involve any eigendecomposition or SVD as in common subspace methods. It is shown that the proposed method is more computationally efficient than a direct ESPRIT algorithm when the ratio of array size to source number is large, at the expense of negligible loss in estimation performance.

\section{Acknowledgement}

The work described in this paper was supported by a grant from City University of Hong Kong (Project No. 7001203).

\section{References}

[1] Y.Hua, T.K.Sarkar and D.D.Weiner, "An L-shaped array for estimating 2-D directions of wave arrival," IEEE Trans. Antennas and Propagation, vol.39, no.2, pp.143-146, 1991

[2] Q.Cheng and Y.Hua, "Further study of the pencil-MUSIC algorithm," IEEE Trans. Aerospace Elect. Syst., vol.32, no.1, pp.284-299, 1996

[3] A.J.van der Veen, P.B.Ober and E.F.Deprettere, "Azimuth and elevation computation in high resolution DOA estimation," IEFE Trans. Signal Processing, vol.40, no.7, pp.1828-1832, 1992

[4] A.L.Swindlehurst and T.Kailath, "Azimuth/elevation direction finding using regular array geometries," IEEE Trans. Aerospace Elect. Syst., vol.29, no.1, pp.145-156, 1993

[5] M.D.Zoltowski, M.Haardt and C.P.Mathews, "Closed-form 2-D angle estimation with rectangular arrays in element space or beamspace via unitary ESPRIT," IEEETrans. Signal Processing, vol.44, no.2, pp.316-328, 1996

[6] T.H.Liu and J.M.Mendel, "Azimuth and elevation direction finding using arbitrary array geometries," IEEETrans. Signal Processing, vol.46, no.7,pp.2061-2065, 1998

[7] V.S.Kedia and B.Chandna, "A new algorithm for 2-D DOA estimation," Signal Processing, vol.60, pp.325-332, 1997

[8] S.Marcos, A.Marsal and M.Benidir, "The propagator method for source bearing estimation," Signal Processing, vol.42, pp.121-138, 1995

[9] P.Li, B.Yu and J.Sun, "A new method for two-dimensional array signal processing in unknown noise environments," Signal Processing, vol.47, pp.319-327, 1995

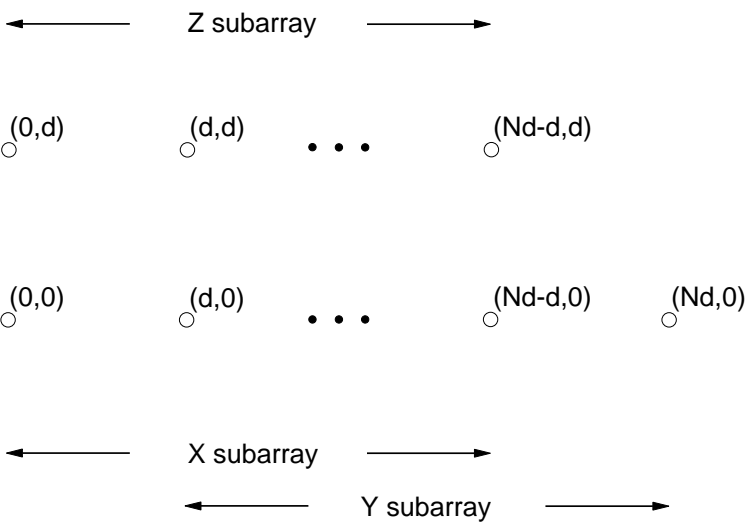

Figure 1: Illustration of the array geometry 


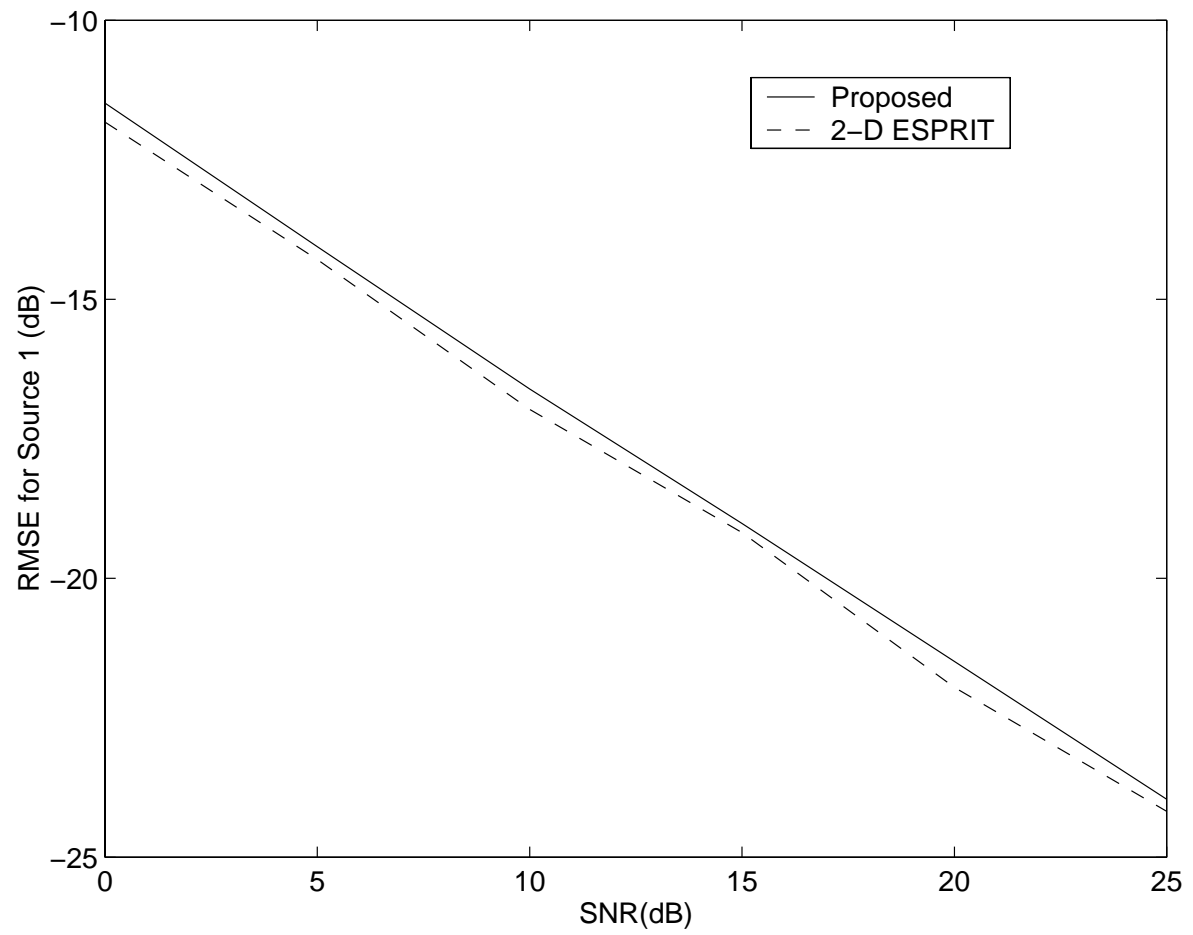

Figure 2: RMSE for the first source versus SNR

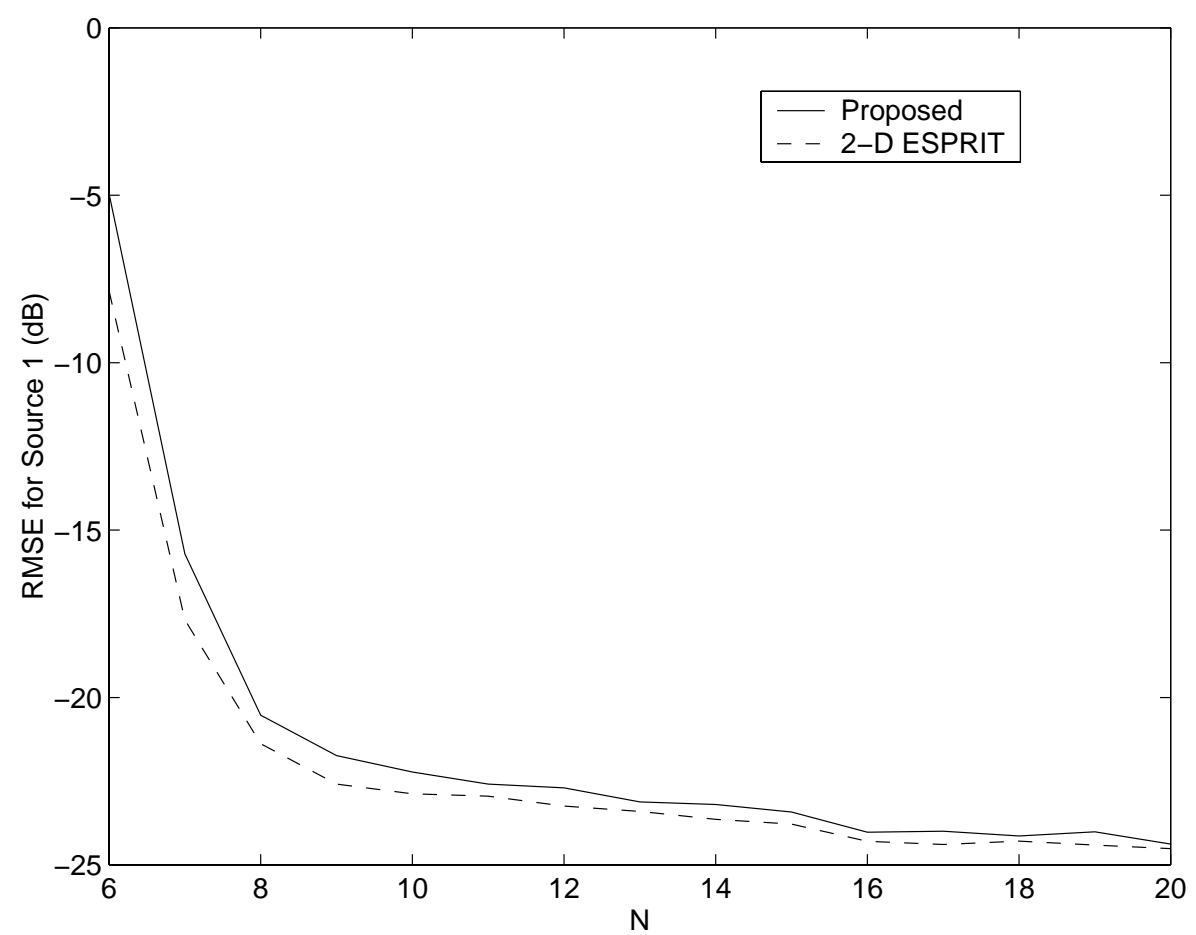

Figure 3: RMSE for the first source versus $N$ at $\mathrm{SNR}=20 \mathrm{~dB}$ 